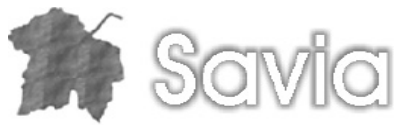

\section{La hermenéutica desde el trabajo social}

\author{
Aurora Zavala Caudillo ${ }^{1}$
}

\section{Resumen:}

La hermenéutica es una teoría poco abordada por el Trabajo Social para el estudio de los fenómenos sociales. Es a partir de los años 90, en la línea del trabajo social latinoamericano, que da inicio el debate a esta postura teórica. El giro cultural incorpora una visión del mundo que permite comprender e interpretar el sentido de acción de los sujetos en el mundo cotidiano, cuya metodología toma distancia con el trabajo social "tradicional". El reto para esta disciplina es abordar orientaciones de análisis sustentadas en una posición teórica que incorpore elementos objetivos y subjetivos de lo social, cuyas características, aportes y limitaciones se analizan en este trabajo.

\begin{abstract}
Hermeneutics is a theory that received little attention from social work to the study of social phenomena. It is from the 90s, along the lines of social work in Latin America, which kicks off the debate at this theoretical position. The cultural turn incorporates a world view that allows understanding and interpreting the meaning of action of the subjects in the everyday world, which methodology takes away with social work "traditional". The challenge for this discipline is to address guidelines for analysis based on a position theory to incorporate objective and subjective elements of the social characteristics, contributions and limitations are discussed in this work.
\end{abstract}

\section{Introducción}

El presente artículo forma parte del trabajo de investigación "Las ciencias de la interpretación y el trabajo social", el cual apunta a la necesidad de reflexionar sobre los enfoques teóricos que permiten dar cuenta de los fenómenos sociales. A continuación usted podrá encontrar las premisas fundamentales que dan sustento a la propuesta metodológica para el trabajo social comunitario. Inicio con un breve recorrido que visualiza los momentos históricos del trabajo social, continúo con la hermenéutica y su contribución al trabajo social, en éste explicito las categorías que constituyen el aporte para nuestra disciplina; planteo redefinir el concepto de comunidad, el sujeto de conocimiento y acción social en dos dimensiones relacionales, la subjetiva y objetiva. Esto me permite enmarcar la propuesta metodológica para el trabajo social comunitario donde se incorpora la visión objetiva y subjetiva de la construcción de la realidad social, a partir de argumentos teóricos para el quehacer profesional y disciplinar del trabajo social en contextos contemporáneos.

\section{De la asistencia y re-conceptualización a lo contemporáneo}

La historia del trabajo social ha pasado por diversas etapas, las cuales dan cuenta de una posición teórica y maneras de abordar el objeto de estudio. Sin duda el trabajo de Mary Richmond (1917) logró sentar los fundamentos de nuestra disciplina. En 1917 logró sentar los fundamentos de nuestra disciplina. En 1917 publica el diagnóstico social, la metodología encuentra el sustento teórico en la medicina y la psiquiatría. La influencia por los procesos históricos, económicos y sociales perfilan el eje de estudio desde una visión lineal de causa-efecto-solución. Por tanto, la realidad se estudia como un hecho social cosificado y homogéneo, donde se pretende dar explicaciones a problemas sociales con el fin de descubrir las causas y efectos que la originan. Este período recibe una fuerte crítica por autores como Vélez (2003) al poner en cuestión la autonomía de las técnicas de investigación e intervención social y la ausencia de un aparato teórico el cual fundamente la práctica profesional. En la década de los 70, da inicio la re-conceptualización; ésta toma distancia con el modelo tradicional, al cuestionar el ejercicio profesional del trabajo social. El estudio de la realidad social se estructura bajo nuevas corrientes teóricas y el hombre es visto como un ser bio-psicosocial; es decir se considera al hombre como un ser integral, donde las relaciones sociales, clase, roles y género apuntan como categorías de análisis. Algunas de sus máximas premisas las constituyen los intentos por crear una teoría del trabajo social donde se incorpora el método científico por oposición a la imitación de una metodología sin ser razonada y cuestionada. 

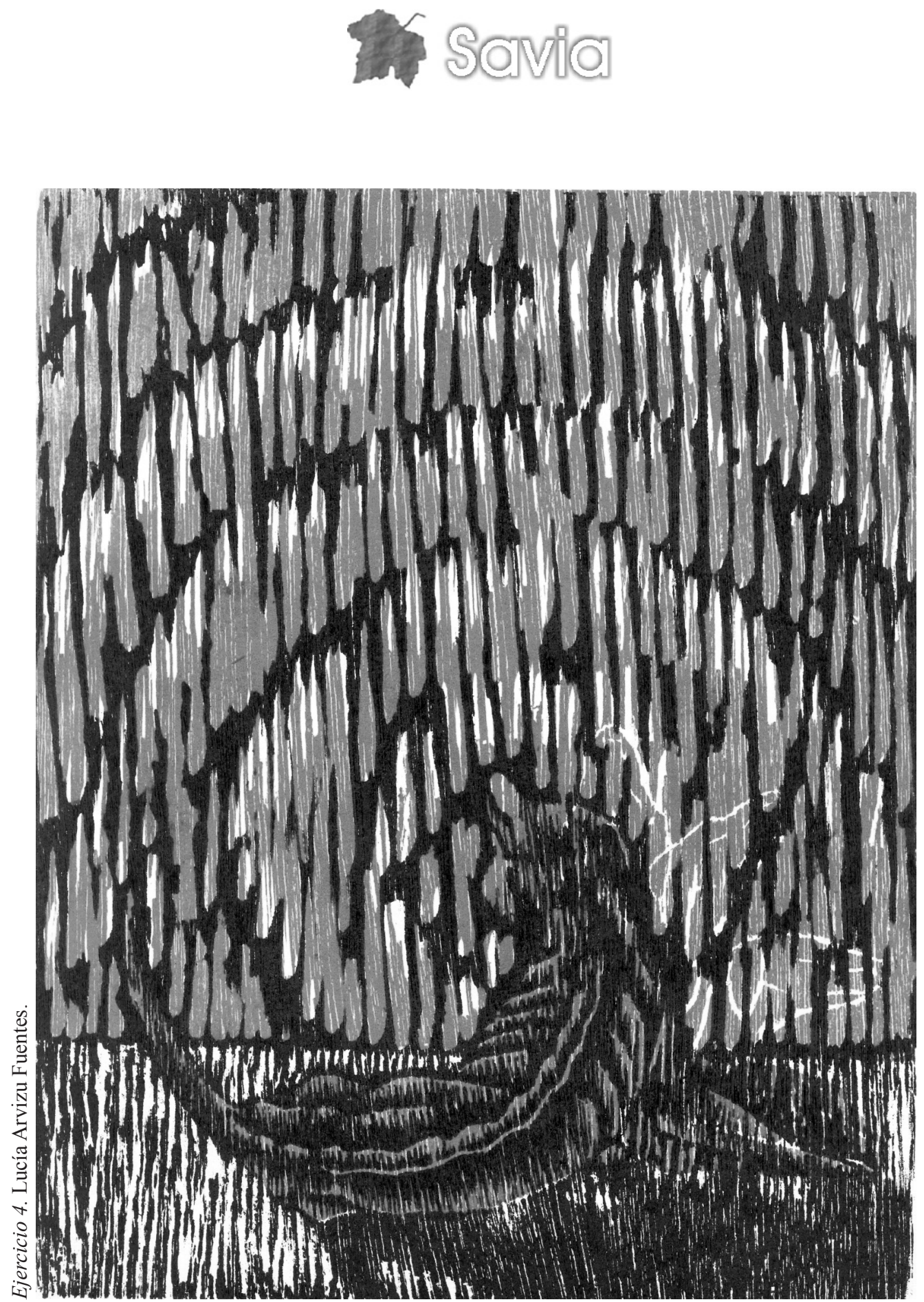

El camino trazado a partir de la re-conceptualización, proporciona argumentos que permiten vislumbrar el cuestionamiento de la metodología, no obstante la tradición vista como un depósito de ideas y conjunto de prácticas seguidas por la imitación o la rutina conlleva sólo a la repetición y anulación de la historia.

A partir de la década de los 80 , se manifiesta la crisis de las Ciencias Sociales, al poner en cuestionamiento los lineamientos del positivismo, que concibe la realidad como única, verdadera y verificable, donde el investigador social se coloca ajeno y externo a la realidad. Como respuesta a la crisis del pensamiento dominante surgen una serie de revoluciones científicas (Kuhn. 2005, las cuales pretenden dar el giro hermenéutico al concebir la realidad compleja, diversa y heterogénea. El retorno del sujeto visto en sus dos dimensiones, objetivo y subjetivo configuran el estudio de los problemas sociales. Bajo este esquema Vélez (2003), plantea los Modelos Contemporáneos en trabajo social. $\mathrm{Su}$ característica se basa en las interacciones de los sujetos con su entorno social desde una perspectivas integral, holística y global. El marco teórico del cual parten son Interaccionismo Simbólico, Fenomenología, Construccionismo, Hermenéutica, Teoría de la 
Complejidad y la Cibernética de Segundo Orden. Colocan la atención en la subjetividad, emociones, vivencias y experiencias; así como en la reflexividad, en la interacción y en la percepción de los sujetos como constitutivos de los social; de esta manera enfatizan los procesos de comunicación a partir del lenguaje, y además configuran la acción social. La apuesta está en mirar a la tradición como un camino que permite la resignificación de las categorías de análisis empleadas con un carácter reflexivo, que dé pauta al examen y reformulación constante de la práctica constante de la práctica profesional, al superar y redefinir los paradigmas de las ciencias que han permeado al Trabajo Social.

\section{La hermenéutica y su contribución para Trabajo Social}

La hermeneuinm arte o técnica del hermenutés o interprete, alude al mito de Hermes, dios de las fronteras y de los viajes, hijo de Zeus; su principal misión consistía en llevar a los dioses de los infiernos los mensajes. Su tarea es traducir e interpretar aquello que no es inteligible en legible, para plasmar la expresión cultural de los hombres.

La hermenéutica en contextos actuales es una corriente filosófica que surge a mediados del siglo XX. Bengoa (2002:44), considera que sus raíces se ubican en Husserl al plantear que es una filosofía, un enfoque y un método, pues enfatiza la reflexión para describir y clarificar la experiencia vivida tratando de introducirse en la dinámica y contenido del actor social. De esta manera, la hermenéutica se inscribe en un paradigma interpretativo, el cual define a la sociedad como una realidad que se construye y mantiene a través de interacciones simbólicas y pautas de comportamiento. La realidad tiene un carácter objetivo y subjetivo, y pretende comprender e interpretar las acciones sociales llenas de significados. Su fin es la captación y reconstrucción de significados. El lenguaje que emplea es metafórico y conceptual, el modo de captar la información y evidencias sociales no se encuentra estructurado, es más bien flexible y desestructurado, el procedimiento es inductivo y la orientación de sus estudios es holístico. La hermenéutica según Beuchot (2009:18), parte de docens y utens. La primera se refiere a una doctrina o teoría general de interpretar, y la segunda a los instrumentos que permiten el estudio teórico para ser aplicados en la práctica.
Es necesario definir los rasgos que precisan la interpretación con la que diseñó la propuesta metodológica; primero la realidad (Berger y Luckman, 2006: 11-14) se constituye por una cualidad propia de los fenómenos que reconocemos, de tal manera que cuando nosotros nos enfrentamos al mundo social, existen estructuras ya determinadas "dadas" como tales. Sin embargo, el hombre tiene la capacidad de cuestionar, mantener o re-significar esa realidad que se presenta objetivada con base a la carga de significados que elabora.

La vida cotidiana se encuentra inmersa en la realidad; ésta es interpretada por los hombres desde su pensamiento, sentido común, acciones y significados. Se visualiza dese una diversidad de capas de experiencia, la cual se ordena a partir de la historicidad, donde la memoria permite traer del pasado al presente fenómenos de esa realidad. Constituye un eje que da la posibilidad de un sentido de orientación en mi presente proyectando el futuro. Para Marx Weber la acción social es toda conducta humana que el sujeto a los sujetos de la acción vinculan con un sentido subjetivo.

La acción social, por tanto (Weber citado en Aguayo, 2006:50) es "una acción en donde el sentido mentado por su sujeto o sujetos está referido a la conducta de otros, orientándose por ésta en su desarrollo". Se enmarca en tres términos decisivos, verstehen, deuten y erkläre. Es decir, comprender e interpretar significaciones a partir de organizar en conceptos el sentido subjetivo y explicar las regularidades de la conducta.

La acción para Schutz (2003:22) es definida como toda conducta humana que es ideada o tramada por el actor social, así la acción, así la acción se proyecta y manifiesta. La acción como proceso en curso se entiende como una actuación en movimiento, adopta una actitud reflexiva sobre mi acción vivo mi presente, lo que ocasiona una determinada experiencia.

Este marco permite interpretar y comprender la serie de acciones sociales en dos planos: primero, al ser interiorizado por los actores con base en las percepciones, ideas y juicios que tienen del mundo, y segundo, que se ubica en el plano objetivo, el cual es materializado por las acciones cotidianas y artefactos simbólicos, en relación de las experiencias y sentido común del actor en constante interacción.

Por tanto, la interpretación siguiendo a Gadamer (2006:371-372), no se limita sólo a los textos y a la comprensión de los fenómenos históricos, involucra 


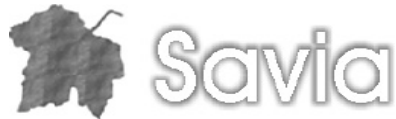

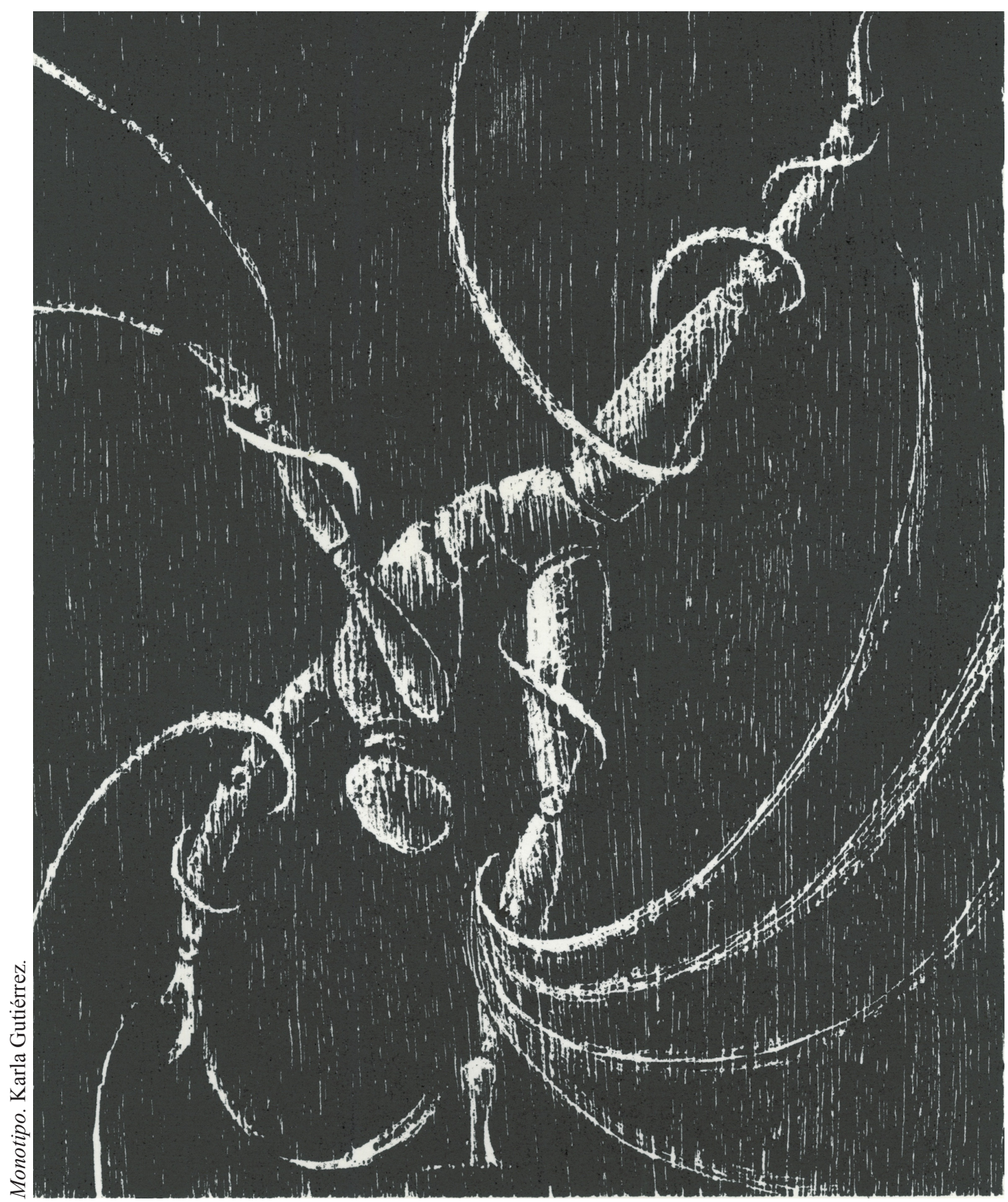

todas las estructuras de sentido concebidas en el arte, religión, ciencia, lenguaje, y todas aquellas motivaciones conscientes e inconscientes de la acción son susceptibles de ser interpretadas.

Las acciones se encuentran inmersas en una trama de significados y artefactos simbólicos, entendiendo por símbolo la estructura de imaginación que devela objetos externos, no sólo es la representación de lo "dado", sino que abre un sinfín de posibilidades de imaginar, crear y pensar. El signo es arbitrario y alude a la configuración de lo "dado", es algo indicado. Las acciones sociales se encuentran inmersas en una telaraña de significados.

La hermenéutica plantea:

- La captación de significados.

- El investigador es partícipe de la realidad estudiada.

- La ciencia y la experiencia no se contraponen en el proceso de investigación.

- Descripción densa de la cultura. 
Hasta aquí presento los principales postulados del planteamiento hermenéutico que retomo. A continuación veremos la propuesta metodológica que nos proporciona este paradigma para el Trabajo Social.

\section{Propuesta metodológica}

La metodología se describe como todo proceso que accede a la transformación de la realidad en datos empíricos aprehensibles y cognoscibles, que buscan develar una determinada parcela de esa realidad. Para ello, el trabajador social debe asumir una actitud metodológica, en términos de Schutz (2006:93-105), la cual nos coloca como "forasteros"3. Esta actitud permite orientar los primeros acercamientos al estudio de la práctica, en tanto en ella se observa, describe e interpreta para la acción social. Desde esta visión teórica, el trabajo social comunitario permite el estudio de los actores desde una dimensión cultural, al mantener, producir y transformar diversos significados según tiempo y contexto. La mirada que asume el trabajador social ${ }^{4}$ para el análisis de la realidad social, es de sujeto a sujeto donde el actor es considerado como un ser simbólico. Por tanto, la realidad social se construye a partir de los mismos sujetos.

Bajo este esquema defino a la comunidad como una categoría de análisis que se genera en un espacio incesante de producciones simbólicas, no sólo de elaboración y expresión de afectividad; sino como un lugar imaginado y sensible de contradicciones. Es ahí donde ocurren fenómenos expresivos que entran en constante tensión y conflicto a partir del reconocimiento y diferencia de experiencias cotidianas. Planteo que el sujeto de conocimiento y de acción social es aquél inmerso en su cotidianidad; por otro lado, la dimensión subjetiva son las ideas, sentimientos, sentido común, representaciones que elaboran los sujetos, y la dimensión objetiva la constituyen los aspectos que se materializan a partir del sentido de la acción y la experiencia vivida, tales como el lenguaje y prácticas cotidianas. Asumir que el estudio de la realidad social es diverso y las maneras de abordarlo dependen de una posición ontológica, epistemológica, teórico metodológica es cumplir con el criterio de vigilancia epistémica y responder a los procesos sociales y culturales que se viven en un mundo contemporáneo.

\section{Reflexiones finales}

La hermenéutica reconoce el papel de la historia; su fin no es anular la tradición histórica, en este caso de la disciplina del Trabajo Social; lo que se pretende es la reflexión crítica con el fin de superar las categorías de análisis utilizadas a partir de repensar y resignificar el ejercicio disciplinar.

El acercamiento a la realidad social no debe limitarse a un solo enfoque paradigmático. Debe de existir la apertura a otras miradas y enfoques de investigación que permitan la comprensión y el conocimiento de la realidad social desde el Trabajo Social, asumiendo con ello la vigilancia epistemológica. En tanto, los contextos de la realidad son diversos, en ellos el tiempo y espacio juegan un papel fundamental en la construcción y comprensión de la realidad social.

La hermenéutica desde el Trabajo Social permite visualizar a los actores sociales inmersos en procesos identitarios y de interacción, donde los ámbitos de acción, en los cuales los ámbitos de acción social se ubican a nivel intragrupal referido referido a un nosotros y el intergrupal a partir de las alianzas y oposiciones. Los escenarios de la acción social se encuentran delimitados desde lo individual, comunidad, región, instituciones; cada uno de estos espacios se subdivide en categorías espaciales. Lo que llamamos objetivos y fines se entienden a la interacción de los actores en relaciones de oposición y ayuda mutua; los discursos sociales posibilitan realizar el análisis de la representación sobre diferentes objetos sociales de los actores investigados.

\section{Bibliografía}

Aguayo, C. (2006). Las profesiones modernas: dilemas del conocimiento y poder. Universidad Tecnológica Metropolitana. Chile. p. 169.

Bengoa Ruiz de Azúa, J. De Heidegger a Habermas. Hermenéutica y fundamentación última en la filosofia contemporánea. Herder. Barcelona. p. 211.

Beuchot, M. (2009). Tratado de hermenéutica analógica. (2002). Hacia un nuevo modelo de interpretación. FFyL UNAM-ITACA. México. p. 237.

Berger y Luckman. (2006). La construcción de la realidad social. Amorrortu. Argentina. p. 233.

Hans-Georg, G. (2006). Verdad y método II. Sígueme. Salamanca, España. p. 429. 


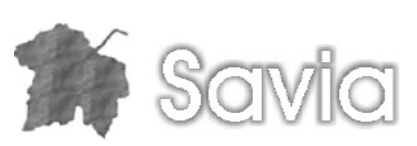

Kuhn, T. S. (2005). La estructura de las revoluciones cientificas. Fondo de Cultura Económica.

Schutz, A. (2003). El problema de la realidad social. Escritos I. Amorrortu. Argentina. p. 326.

(2006). El forastero, ensayo de psicología social. En estudio sobre teoría social. Amorrortu. Argentina. p. 276.

Vélez Restrepo, O. (2003). Reconfigurando el trabajo social, perspectivas y tendencias contemporáneas. Espacio. Buenos Aires. p. 160.

\section{Notas al pie}

1 Docente de la Escuela Nacional de Trabajo Social de la UNAM. Correo electrónico.auro_28@yahoo.com.mx

2 Trabajo de investigación de la práctica comunitaria, en colaboración con el Maestro Martín Sánchez Villal, docente de la Escuela Nacional de Trabajo Social de la UNAM.

3 Indica la actitud que debe de asumir todo investigador de lo social. Perfila su papel de hombre de ciencia, el cual observa, describe, interpreta y conoce el mundo social con la mayor claridad posible, de acuerdo con los ideales y coherencia metodológica.

4 Cuando hablo de la mirada refiero a la postura ontológica, epistemológica que asume el trabajador social para el análisis de los fenómenos sociales. 